\title{
Prognostic Value and Optimal Candidates of Primary Tumor Surgery in Metastatic Adenoid Cystic Carcinoma of the Head and Neck: First Evidence from a Population-Based Study
}

\section{Di Zhang ( $\nabla$ irisaaron@163.com)}

Chinese Academy of Medical Sciences and Peking Union Medical College

\section{Research}

Keywords: adenoid cystic carcinoma, survival, metastasis, prognosis, surveillance, epidemiology, and end results (SEER) database

Posted Date: November 10th, 2021

DOI: https://doi.org/10.21203/rs.3.rs-1042947/v1

License: (9) This work is licensed under a Creative Commons Attribution 4.0 International License. Read Full License 


\section{Abstract}

Objective: The prognostic value of primary tumor surgery (PTS) for patients with metastatic adenoid cystic carcinoma of the head and neck (HNACC) at initial diagnosis has never been studied. The aims of this study were to determine the prognostic significance and identify optimal candidates of PTS.

Materials and Methods: Patients with metastatic HNACC (stage IVC) at initial diagnosis were identified from the Surveillance, Epidemiology and End Results (SEER) database between 2004 and 2015. Correlated variables, including age, sex, race, tumor stage, metastatic site, treatment, and survival information were extracted. Kaplan-Meier analysis, log-rank test, and multivariate Cox regression model were performed to evaluate the impact of PTS on survival outcomes, including overall survival (OS) and cancer-specific survival (CSS).

Results: Overall, 155 patients were identified from database. Of those, $93(60 \%)$ patients underwent palliative PTS. Patients with lung metastasis alone were more likely undergo PTS (c2=15.042, $P=0.002$ ). The univariate analysis showed that PTS were associated with significantly improved survival than noPTS ( $P=0.001$ for CSS; $P \otimes 0.001$ for OS). The median CSS for patients who received PTS and those who did not receive PTS were 64.0 and 22.0 months, respectively. The median OS for patients who received PTS and those without PTS were 43.0 and 16.0 months, respectively. Notably, in multivariate model, the OS of patients who underwent PTS was better than that of patients who did not (HR=0.586, $P=0.017)$, but there was no significantly improvement in CSS. In addition, subgroup analyses further revealed that patients with T3-4 or N0 stage might benefit from PTS.

Conclusion: PTS significantly improved OS in patients with metastatic HNACC. Besides, PTS had a favorable prognostic impact on highly-selected patients, namely T3-4 and NO stage, which could be adopted in future clinical practice. Further multicenter prospective studies are still needed to verify these outcomes.

\section{Introduction}

Adenoid cystic carcinoma (ACC) is a relatively rare malignancy that arises mostly in salivary glands(1). ACC accounts for approximately $25 \%$ of all salivary gland carcinomas, and only $1 \%$ of all head and neck malignancies(2). Adenoid cystic carcinoma of the head and neck (HNACC) is a generally slow-growing but highly aggressive malignant tumor, which has the characteristics of local recurrence and distant metastasis $(3,4)$. Once metastatic disease is present, the median overall survival (OS) of patients with salivary ACC is about three years $(5,6)$. Among them, up to $10 \%$ of patients can survive for more than 10 years due to indolent tumor growth $(7,8)$. However, one-third of patients may develop more rapid disease progression and die within 2 years after metastasis $(8,9)$. Although chemotherapy, expectant treatment, selective metastatic resection and optimal supportive treatment can be used for the treatment of metastatic HNACC $(10)$, the survival benefit is very limited $(11,12)$. Thus, more attention should be paid to those patients with distant metastasis. 
Currently, accumulating studies have reported the favorable prognostic impact of primary tumor surgery (PTS) on multiple metastatic cancers, such as breast cancer, gastric cancer, and colorectal cancer(1315), but there was no relevant study on PTS for metastatic HNACC. In the up-to-date ASCO guideline, for ACC and/or low-grade tumors with indolent biology with limited metastases, local ablative treatments (such as surgery or stereotactic body radiation therapy) may be offered to delay local disease progression(16). Nevertheless, the guideline did not specify whether surgical management of primary site should be performed. It was still unknown whether the removal of the primary tumor could lead to survival benefits for patients with metastatic HNACC.

Therefore, we conducted a population-based study to determine the prognostic significance and identify optimal candidates of PTS by using Surveillance, Epidemiology, and End Results (SEER) program databases.

\section{Method And Meterial}

\section{Data Collection}

The data was extracted from the SEER database, including additional treatment information, using SEER ${ }^{\star}$ Stat software (version 8.3.5). ACC as defined by the World Health Organization (WHO) 2008 salivary gland tumor classification, based on the International Classification of Diseases for Oncology, 3rd ed. (ICD-0-3) system (ACC = 8200). Since the American Joint Committee on Cancer (AJCC) 8th edition is not accessible in the SEER registry, we considered data on ACC cases that were staged using the AJCC 6th and 7th editions, effectively 2004 and onward. The 7th edition has not undergone major revisions, so the cases classified according to the 6th and 7th editions of AJCC have been merged.

The inclusion criteria were listed as follows: (1) pathologically confirmed ACC; (2) had distant metastasis at initial diagnosis; (3) diagnosed between 2004 and 2015, because several essential factors were only available for patients diagnosed after 2004; (4) had completed follow-up and survival information. Patients were excluded in our study if the following criteria were met: (1) ACC at anatomic sites other than head and neck; (2) no clear derived AJCC TNM staging, such as Tx, Nx and Mx stage. Information used in our study was based on the most recent follow-up data available (December 31, 2018).

Baseline characteristics including demographics, tumor characteristic, and treatment were collected. The relative covariates were retrieved as follows: patient ID, age at diagnosis, sex, race, primary tumor site, histological grade, derived AJCC T staging, N staging, metastatic sites, and treatment. In our study, PTS represented total or at least partial excision of the primary tumor with or without adjacent site. Local destructive therapies, such as excisional biopsy, electrocautery and cryosurgery, were excluded from the definition of PTS. In addition, the pathological grade was expressed as grade I, II, III and IV. Among them, grade I and grade II were defined as well differentiated and moderately differentiated, respectively. Grade III represented poorly differentiated, and grade IV represented undifferentiated or anaplastic. 
This study was deemed exempt from the approval of the medical ethics committee of the National Cancer Center/Cancer Hospital Chinese Academy of Medical Sciences, because the data were from a public health database accessible to applicants.

\section{Statistical Analysis}

OS was defined as the time from initial diagnosis to overall mortality. Cancer-specific survival (CSS) was calculated as the time from initial diagnosis to the date of cancer specific death. Statistical analyses were performed using SPSS statistical software version 23.0 (IBM Corp., Armonk, NY, United States). Differences in demographics and clinical characteristics were assessed with chi-square test or t-test. Univariate survival analysis was performed using the Kaplan-Meier method and the difference in survival curves between the two groups was estimated by the log-rank test. Figures were produced using GraphPad Prism software version 9.0 (GraphPad Software, Inc., United States). Multivariate analysis was performed by a Cox proportional hazards regression model to evaluate the independent prognostic factors associated with improved survival. Forest plots summarizing hazards ratios (HR) for PTR versus no-PTR in the subgroup analyses were performed by RStudio (Version 1.4.1717@2009-2021 RStudio, PBC). For all statistical tests, significance was considered to be achieved when $P<0.05$.

\section{Result}

\section{Baseline Characteristics}

A total of 155 patients with metastatic HNACC at initial diagnosis were identified from the SEER database between 2004 and 2015. The median age at diagnosis was 62 years (range: 20-93). More than half $(n=81,52.3 \%)$ of the patients were female. The majority of patients were white race $(n=121,78.1 \%)$. Major salivary glands ( $n=83,53.5 \%$ ) were the most common site of primary lesions, including parotid gland $(n=35)$, submandibular gland $(n=35)$, sublingual gland $(n=4)$ and major salivary gland, NOS $(n=9)$. Among patients with a clear pathological grade, the number of patients with grade I-II was roughly equal to that of patients with grade III-IV. Moreover, 118 (76\%) patients had T3-4 stage HNACC, and nearly half ( $\mathrm{n}=67$, $43.2 \%$ ) had regional lymph node metastasis at the time of initial diagnosis. Among patients with clear metastatic sites, lung $(n=63,40.6 \%)$, bone $(n=22,14.2 \%)$ and liver $(n=11,11.0 \%)$ were the most common metastatic sites, and only one patient had brain metastasis. In terms of treatment, 93 (60.0\%) patients received PTS, whereas 62 patients $(40.0 \%)$ did not undergo PTS. Among 67 patients with regional lymph node metastasis, nearly half $(n=34,50.7 \%)$ patients received regional lymph nodes removed. None of the metastases were given surgery. In addition, $39.4 \%(n=61)$ and $38.1 \%(n=59)$ of patients received radiotherapy and chemotherapy, respectively.

Baseline characteristics of the PTS and no-PTS group were presented in Table 1. Significant differences were observed in grade, metastasis sites, radiotherapy and chemotherapy (All $P<0.05$ ). Patients with lung metastasis alone were more likely undergo PTS $(\chi 2=15.042, P=0.002)$. For patients who received PTS, the proportion of patients who received radiotherapy reached $63.4 \%$, which was much higher than that of patients who did not undergo PTS $(\chi 2=56.514, P<0.001)$. On the contrary, patients receiving 
chemotherapy were more common in those who did not undergo PTS ( $50.0 \%$ vs $30.1 \%, \chi 2=56.514$, $P=0.012)$. 
Table 1

Baseline characteristic of metastatic HNACC patients at initial diagnosis.

\begin{tabular}{|c|c|c|c|c|c|}
\hline Characteristic & Total $(n=155)$ & $\begin{array}{l}\text { PTS } \\
(n=93)\end{array}$ & $\begin{array}{l}\text { No-PTS } \\
(n=62)\end{array}$ & $x^{2}$ & $P$ \\
\hline Gender & & & & 1.246 & 0.264 \\
\hline Male & $74(47.7)$ & $41(44.1)$ & $33(53.2)$ & & \\
\hline Female & $81(52.3)$ & $52(55.9)$ & $29(46.8)$ & & \\
\hline Age at diagnosis & & & & 0.728 & 0.393 \\
\hline$<60$ & $74(47.7)$ & $47(50.5)$ & $27(43.5)$ & & \\
\hline$\geq 60$ & $81(52.3)$ & $46(49.5)$ & $35(56.5)$ & & \\
\hline Race & & & & 2.982 & 0.225 \\
\hline White & 121(78.1) & 76(81.7) & $45(72.6)$ & & \\
\hline Black & 21(13.5) & $9(9.7)$ & 12(19.4) & & \\
\hline Others & $13(8.4)$ & $8(8.6)$ & $5(8.1)$ & & \\
\hline Primary site & & & & 1.107 & 0.293 \\
\hline Major salivary glands & $83(53.5)$ & $53(57)$ & $30(48.4)$ & & \\
\hline Minor salivary glands & $72(46.5)$ & $40(43)$ & $32(51.6)$ & & \\
\hline Grade & & & & 27.152 & $<0.001$ \\
\hline$|-| \mid$ & $33(21.3)$ & 31(33.3) & $2(3.2)$ & & \\
\hline III-IV & $31(20)$ & $22(23.7)$ & $9(14.5)$ & & \\
\hline Unknown & $91(58.7)$ & $40(43)$ & $51(82.3)$ & & \\
\hline T stage & & & & 0.095 & 0.758 \\
\hline T1-2 & $37(23.9)$ & $23(24.7)$ & 14(22.6) & & \\
\hline T3-4 & 118(76.1) & 70(75.3) & 48(77.4) & & \\
\hline N stage & & & & 1.932 & 0.165 \\
\hline NO & $88(56.8)$ & $57(61.3)$ & $31(50)$ & & \\
\hline N1-3 & $67(43.2)$ & $36(38.7)$ & $31(50)$ & & \\
\hline Metastasis sites & & & & 15.042 & 0.002 \\
\hline Lung alone & 49(31.6) & $37(39.8)$ & 12(19.4) & & \\
\hline Lung and other sites & $14(9)$ & $4(4.3)$ & 10(16.1) & & \\
\hline
\end{tabular}




\begin{tabular}{|llllll|}
\hline Characteristic & Total (n=155) & $\begin{array}{l}\text { PTS } \\
(\mathbf{n = 9 3})\end{array}$ & $\begin{array}{l}\text { No-PTS } \\
(\mathbf{n}=62)\end{array}$ & $\mathbf{X}^{2}$ & $\boldsymbol{P}$ \\
\hline Other sites & $25(16.1)$ & $10(10.8)$ & $15(24.2)$ & & \\
\hline Unknown & $67(43.2)$ & $42(45.2)$ & $25(40.3)$ & & \\
\hline Radiotherapy & & & & 56.514 & $<0.001$ \\
\hline Yes & $61(39.4)$ & $59(63.4)$ & $2(3.2)$ & & \\
\hline No/unknown & $94(60.6)$ & $34(36.6)$ & $60(96.8)$ & & \\
\hline Chemotherapy & & & & 6.244 & 0.012 \\
\hline Yes & $59(38.1)$ & $28(30.1)$ & $31(50)$ & & \\
\hline No & $96(61.9)$ & $65(69.9)$ & $31(50)$ & & \\
\hline Endpoint event & & & & 6.523 & 0.038 \\
\hline Alive & $48(31)$ & $36(38.7)$ & $12(19.4)$ & & \\
\hline Dead attributable to ACC & $81(52.3)$ & $43(46.2)$ & $38(61.3)$ & & \\
\hline Death of other cause & $26(16.8)$ & $14(15.1)$ & $12(19.4)$ & & \\
\hline
\end{tabular}

\section{Survival Outcome}

The median follow-up time was 26.0 months (range 2-140), 81 patients (52.3\%) died of ACC-specific causes, and 26 patients (16.8\%) died of other cause. The survival analysis by Kaplan-Meier estimates and Log-Rank tests showed that PTS was significantly associated with better CSS than those patients without PTS (64.0 months vs. 22.0 months, $\chi 2=11.210, P=0.001$ ) (Figure $1 A$ ). Similarly, the median OS was significantly improved in patients who received PTS than those who did not (43.0 vs. 16.0 months, $\chi 2=15.039, P<0.001)$ (Figure 1B).

\section{Prognostic Factors}

As shown in Table 2, the clinical characteristics were evaluated to determine their prognostic value for CSS and OS. Univariate analysis showed that grade I-II ( $P=0.001$ for CSS; $P<0.001$ for OS), N0 stage ( $P<0.001$ for CSS; $P=0.001$ for OS), lung metastasis alone ( $P=0.020$ for CSS; $P=0.038$ for OS), radiotherapy ( $P=0.022$ for CSS; $P=0.021$ for OS) and PTS ( $P=0.001$ for CSS; $P<0.001$ for OS) were associated with better CSS and OS. The OS was also improved in patients with T1-2 stage than those with T3-4 stage HNACC $(P=0.048)$. However, age, race, gender, and primary site were not significantly related to CSS and OS. 
Table 2

Univariate analyses of factors influencing CSS and OS in metastatic HNACC

Factors

\section{Gender}

Female vs Male

0.825

$0.531 \sim 1.282$

0.392

0.779

$0.531 \sim 1.143$

0.201

Age at diagnosis

$\begin{array}{lllllll}\geq 60 \text { vs }<60 & 0.964 & 0.623 \sim 1.493 & 0.870 & 1.163 & 0.796 \sim 1.700 & 0.436 \\ \text { Race } & & & & & \end{array}$

Black vs White

$1.182 \quad 0.635 \sim 2.200$

0.598

1.096

$0.631 \sim 1.905$

0.745

Others vs White

1.101

$0.502 \sim 2.415$

0.809

1.056

$0.529 \sim 2.108$

0.877

\section{Primary site}

Major vs Minor salivary glands

\section{Grade}

III-IV vs I-II

Unknown vs I-II

T stage

T3-4 vs T1-2

1.618

$0.922 \sim 2.839$

0.093

1.636

$1.005 \sim 2.663$

0.048

\section{$\mathrm{N}$ stage}

N1-3 vs NO

2.702

$1.714 \sim 4.259$

$<0.001$

1.952

$1.319 \sim 2.888$

0.001

Metastasis sites

Lung and other sites vs Lung alone

Other sites vs Lung alone

Unknown vs Lung alone

\section{Radiotherapy}

Yes vs No/unknown

$0.583 \quad 0.368 \sim 0.925$

0.022

0.627

$0.422 \sim 0.933$

0.021

\section{Chemotherapy}

Yes vs No/unknown

$1.658 \quad 1.066 \sim 2.580$

$\mathbf{0 . 0 2 5} 1.473$

$0.999 \sim 2.171$

0.050 


\section{Surgery of primary site}

Yes vs No/unknown

$$
\begin{array}{llll}
0.481 & 0.309 \sim 0.748 & \mathbf{0 . 0 0 1} & 0.479
\end{array}
$$

$0.326 \sim$

0.703

Table 3

Multivariate analyses of factors influencing CSS and OS in metastatic HNACC.

\begin{tabular}{|lllllll|}
\hline & \multicolumn{5}{c}{ CSS } & \multicolumn{3}{c|}{ OS } & \\
\hline & HR & $95 \%$ Cl & P & HR & $95 \%$ Cl & P \\
\hline Grade & & & 0.001 & & & 0.001 \\
\hline III-IV vs I-II & 5.392 & $2.261 \sim 12.858$ & $<0.001$ & 3.812 & $1.842 \sim 7.890$ & $<0.001$ \\
\hline Unknown vs I-II & 3.497 & $1.585 \sim 7.711$ & 0.002 & 2.286 & $1.158 \sim 4.512$ & 0.017 \\
\hline T stage & & & & & & \\
\hline T3-4 vs T1-2 & 1.830 & $1.030 ~ 3.251$ & 0.039 & 1.946 & $1.177 \sim 3.218$ & 0.009 \\
\hline N stage & & & & & & \\
\hline N1-3 vs N0 & 2.779 & $1.739-4.440$ & $<0.001$ & 1.738 & $1.149 \sim 2.631$ & 0.009 \\
\hline Surgery of primary site & & & & & & \\
\hline Yes vs No & - & - & - & 0.586 & $0.378 \sim 0.910$ & 0.017 \\
\hline Abbreviation: PTS, primary tumor surgery; CSS, cancer-specific survival; OS, overall survival. \\
\hline
\end{tabular}

In the multivariate model, grade, $\mathrm{T}$ stage, $\mathrm{N}$ stage were independent prognostic factors influencing both CSS and OS (All $P<0.05$ ). Grade IIIIIV (HR=5.392, $P<0.001$ for CSS; HR=3.812, $P<0.001$ for OS), T3-4 stage (HR=1.830, $P=0.039$ for CSS; HR=1.946, $P=0.009$ for OS), and N1-3 stage (HR=2.779, $P<0.001$ for CSS; $\mathrm{HR}=1.738, P=0.009$ for OS) were associated with worse CSS and OS after adjusting for other prognostic factors. Notably, PTS was also favorable prognostic factors for OS. The OS of patients who underwent PTS was better than that of patients who did not (HR $=0.586, P=0.017)$, but there was no statistically significant improvement in CSS ( $P>0.05)$.

\section{Subgroup Analysis}

Exploratory subgroup analyses were conducted to explore further assess the prognostic value of PTS. The HRs and $95 \%$ Cls for PTS versus no-PTS of each subgroup were shown in forest plot (Figure 2). Compared with no-PTS, PTS was significantly associated with favorable CSS and OS in the subgroups of patients who had T3-4 stage ( $P<0.001$ for both CSS and OS) and N0 stage ( $P=0.005$ for CSS, $P=0.001$ for OS). Additionally, patients who underwent PTS had significantly improved OS in the subgroups of patients with other sites metastasis $(P=0.046)$. In contrast, PTS was not a significant prognostic factor of 
CSS for patients with other sites metastasis $(P=0.210)$. The Kaplan-Meier curves of CSS and OS in different subgroup were presented in Figure 3.

\section{Discussion}

To our knowledge, this was the first study of the role of PTS in the treatment of metastatic HNACC. It was found that removing the primary tumor without resection of distant metastasis can bring survival benefits for patients with metastatic HNACC. Most importantly, our research has identified the optimal candidates for PTS, which contributes to precision medicine.

In our study, the impact of clinicopathological characteristics and PTS on the survival of metastatic HNACC patients was presented. Patients with grade I-II had significantly better prognosis than those with grade III-IV HNACC ( $P<0.001$ for both CSS and OS). Besides, patients with T1-2 stage $(P=0.039$ for CSS; $P=0.009$ for OS) and absence of lymph node metastasis ( $P<0.001$ for CSS; $P=0.009$ for OS) was associated with better survival. Our results were in accordance with previous retrospective studies on $A C C$, and the grade of pathological differentiation and T stage were independent prognostic factors $(17$, 18). Of particular note was the marked improvement in survival among patients with PTS, which was undoubtedly good news for head and neck surgeons. The median CSS of patients with PTS and no-PTS were 64.0 months and 22.0 months, respectively. The median OS for the PTS group was 43.0 months; and for the no-PTS groups, it was 16.0 months. In multivariate analyses, PTS was not associated with CSS benefits, whereas it does confer increased OS in metastatic HNACC (HR=0.586, $P=0.017)$. Based on the above findings, PTS should be considered for the treatment of metastatic HNACC. Notably, although the therapeutic value of PTS has been proven in HNACC and other types of cancer $(19,20)$, there was no consensus on the underlying mechanism of surgical advantages. It has been reported that surgical resection can decrease the risk of weight loss and nutritional depletion(21), or delay the occurrence of anemia, hypoproteinemia and even cachexia by decreasing the tumor burden(22, 23). However, up till now, these hypotheses have not been validated. From this perspective, the mechanism of the surgeryderived merits deserves further investigation.

The secondary purpose of this study was to identify predictive factors for selecting suitable candidates of PTS, which provided more reliable evidence for head and neck surgeons. In our study, PTS was significantly associated with better OS and CSS in patients with T3-4 or N0 stage (all $P<0.05$ ), which suggested that PTS may be an effective treatment option for patients with the above clinical characteristics. Specifically, patients with positive regional lymph nodes did not benefit significantly from PTS, but for patients without regional lymph nodes metastasis, patients who received PTS had favorable prognosis than those who did not. Even if metastatic disease exists, PTS may be more suitable for patients with relatively indolent diseases. On the other hand, PTS was shown to be of prognostic value for metastatic patients with T3-T4 stage in our study. We speculated that removal of the primary tumor may reduce the tumor burden and control the local tumor-related symptoms to a certain extent, and ultimately translates into significantly survival benefit. Interestingly, our finding was inconsistent with some retrospective studies $(24,25)$. In shi xiao et al research, PTS did not confer a survival benefit in 
patients with T4a-4b stage disease. They considered that aggressive surgical excision was not only unable to bring survival benefits, but was also highly likely to seriously affect the integrity of crucial anatomical structures in the head and neck region $(24,25)$. This phenomenon may be explained by the inconsistency of the enrolled populations between different study. The tumor heterogeneity could not be ignored. Except for ACC, their research also included some other pathological types of salivary gland tumors (the specific number was unknown). Thus, more well-designed studies are needed to validate the above results.

Despite its advantages, our research had some limitations, most of which were inherent in secondary data analysis of this nature. As a retrospective study, there was inevitably inherent selection bias. Other limitations included the lack of information on clinical symptom, number of metastases, surgical margins and perineural invasion status that could have impact on prognosis. In addition, the subsequent treatment of these patients was unknown, due to the limitation of SEER database. Therefore, further prospective randomized studies are needed to provide evidence that whether this population should be treated with PTS.

\section{Conclusion}

In summary, our population-based study for the first time demonstrated the favorable impact of PTS on survival outcomes in patients with metastatic HNACC. Optimal candidates for PTS were identified as T34 and N0 stage disease, while the prognostic efficacy in their counterparts with T1-2 or N1-3 stage requires more evidence. Long-term prospective studies are still awaited to develop more refined selection criteria.

\section{Authors' contributions}

DZ was responsible for collecting the data, analysis, searching the literature, editing the manuscript, and preparation of the manuscript for submission.

\section{Declarations}

\section{Authors' contributions}

DZ was responsible for collecting the data, analysis, searching the literature, editing the manuscript, and preparation of the manuscript for submission.

\section{Acknowledgements:}

NO.

\section{Funding}

Not applicable. 


\section{Ethical approval statement:}

This study was deemed exempt from the approval of the medical ethics committee of the National Cancer Center/Cancer Hospital Chinese Academy of Medical Sciences, because the data were from a public health database accessible to applicants.

\section{Clinical trial registration number (if applicable):}

Not applicable.

\section{Data availability statement}

The data might be made available upon request, and some restrictions will apply.

\section{Conflicts of interest/Competing interests}

NO.

\section{References}

1. Young A, Okuyemi OT. Malignant Salivary Gland Tumors. StatPearls. Treasure Island (FL)2021.

2. Atallah S, Casiraghi O, Fakhry N, Wassef M, Uro-Coste E, Espitalier F, et al. A prospective multicentre REFCOR study of 470 cases of head and neck Adenoid cystic carcinoma: epidemiology and prognostic factors. Eur J Cancer. 2020;130:241-9.

3. Amit M, Binenbaum Y, Sharma K, Ramer N, Ramer I, Agbetoba A, et al. Analysis of failure in patients with adenoid cystic carcinoma of the head and neck. An international collaborative study. Head Neck. 2014;36(7):998-1004.

4. Ali S, Bryant R, Palmer FL, DiLorenzo M, Shah JP, Patel SG, et al. Distant Metastases in Patients with Carcinoma of the Major Salivary Glands. Ann Surg Oncol. 2015;22(12):4014-9.

5. Spiro RH, Huvos AG. Stage means more than grade in adenoid cystic carcinoma. Am J Surg. 1992;164(6):623-8.

6. van der Wal JE, Becking AG, Snow GB, van der Waal I. Distant metastases of adenoid cystic carcinoma of the salivary glands and the value of diagnostic examinations during follow-up. Head Neck. 2002;24(8):779-83.

7. Terhaard CH, Lubsen H, Van der Tweel I, Hilgers FJ, Eijkenboom WM, Marres HA, et al. Salivary gland carcinoma: independent prognostic factors for locoregional control, distant metastases, and overall survival: results of the Dutch head and neck oncology cooperative group. Head Neck. 2004;26(8):681-92; discussion 92-3.

8. Spiro RH. Distant metastasis in adenoid cystic carcinoma of salivary origin. Am J Surg. 1997;174(5):495-8.

9. Laurie SA, Ho AL, Fury MG, Sherman E, Pfister DG. Systemic therapy in the management of metastatic or locally recurrent adenoid cystic carcinoma of the salivary glands: a systematic review. 
Lancet Oncol. 2011;12(8):815-24.

10. Pfister DG, Spencer S, Adelstein D, Adkins D, Anzai Y, Brizel DM, et al. Head and Neck Cancers, Version 2.2020, NCCN Clinical Practice Guidelines in Oncology. J Natl Compr Canc Netw. 2020;18(7):873-98.

11. Lorini L, Ardighieri L, Bozzola A, Romani C, Bignotti E, Buglione M, et al. Prognosis and management of recurrent and/or metastatic head and neck adenoid cystic carcinoma. Oral Oncol. 2021;115:105213.

12. Gao M, Hao Y, Huang MX, Ma DQ, Luo HY, Gao Y, et al. Clinicopathological study of distant metastases of salivary adenoid cystic carcinoma. Int J Oral Maxillofac Surg. 2013;42(8):923-8.

13. Warschkow R, Guller U, Tarantino I, Cerny T, Schmied BM, Thuerlimann B, et al. Improved Survival After Primary Tumor Surgery in Metastatic Breast Cancer: A Propensity-adjusted, Population-based SEER Trend Analysis. Ann Surg. 2016;263(6):1188-98.

14. Warschkow R, Baechtold M, Leung K, Schmied BM, Nussbaum DP, Gloor B, et al. Selective survival advantage associated with primary tumor resection for metastatic gastric cancer in a Western population. Gastric Cancer. 2018;21(2):324-37.

15. Tarantino I, Warschkow R, Worni M, Cerny T, Ulrich A, Schmied BM, et al. Prognostic Relevance of Palliative Primary Tumor Removal in 37,793 Metastatic Colorectal Cancer Patients: A PopulationBased, Propensity Score-Adjusted Trend Analysis. Ann Surg. 2015;262(1):112-20.

16. Geiger JL, Ismaila N, Beadle B, Caudell JJ, Chau N, Deschler D, et al. Management of Salivary Gland Malignancy: ASCO Guideline. J Clin Oncol. 2021;39(17):1909-41.

17. van Weert S, Bloemena E, van der Waal I, de Bree R, Rietveld DH, Kuik JD, et al. Adenoid cystic carcinoma of the head and neck: a single-center analysis of 105 consecutive cases over a 30-year period. Oral Oncol. 2013;49(8):824-9.

18. Shen W, Sakamoto N, Yang L. Model to Predict Cause-Specific Mortality in Patients with Head and Neck Adenoid Cystic Carcinoma: A Competing Risk Analysis. Ann Surg Oncol. 2017;24(8):2129-36.

19. Tierney JF, Chivukula SV, Wang X, Pappas SG, Schadde E, Hertl M, et al. Resection of primary tumor may prolong survival in metastatic gastroenteropancreatic neuroendocrine tumors. Surgery. 2019;165(3):644-51.

20. Zheng M, Li Y, Li T, Zhang L, Zhou L. Resection of the primary tumor improves survival in patients with gastro-entero-pancreatic neuroendocrine neoplasms with liver metastases: A SEER-based analysis. Cancer Med. 2019;8(11):5128-36.

21. Cook AD, Single R, McCahill LE. Surgical resection of primary tumors in patients who present with stage IV colorectal cancer: an analysis of surveillance, epidemiology, and end results data, 1988 to 2000. Ann Surg Oncol. 2005;12(8):637-45.

22. Turner N, Tran B, Tran PV, Sinnathamby M, Wong HL, Jones I, et al. Primary Tumor Resection in Patients With Metastatic Colorectal Cancer Is Associated With Reversal of Systemic Inflammation and Improved Survival. Clin Colorectal Cancer. 2015;14(3):185-91. 
23. Pockaj BA, Wasif N, Dueck AC, Wigle DA, Boughey JC, Degnim AC, et al. Metastasectomy and surgical resection of the primary tumor in patients with stage IV breast cancer: time for a second look? Ann Surg Oncol. 2010;17(9):2419-26.

24. Shi X, Dong F, Wei W, Song K, Huang N, Lu Z, et al. Prognostic significance and optimal candidates of primary tumor resection in major salivary gland carcinoma patients with distant metastases at initial presentation: A population-based study. Oral Oncol. 2018;78:87-93.

25. Shi X, Huang NS, Shi RL, Wei WJ, Wang YL, Ji QH. Prognostic value of primary tumor surgery in minor salivary-gland carcinoma patients with distant metastases at diagnosis: first evidence from a SEER-based study. Cancer Manag Res. 2018;10:2163-72. 